\title{
Association between phosphorus intake and bone health in the NHANES population
}

\author{
Albert W Lee ${ }^{1}$ and Susan S Cho ${ }^{2^{*}}$
}

\begin{abstract}
The objective of this study was to estimate the independent associations between intake of phosphorus $(\mathrm{P})$ and bone health parameters such as bone mineral content (BMC) and bone mineral density (BMD). It provides odds ratio (OR) of osteoporosis with quartiles of $\mathrm{P}$ intake adjusted for covariates (i.e., age, gender, $\mathrm{BMI}$, and consumption of calcium (Ca), protein, total dairy foods, and vitamin D as well as intakes of supplemental Ca, vitamin D, and multivitamins/minerals). Data came from males and females aged 13-99 years who participated in the 2005-2010 National Health and Nutrition Examination Survey (NHANES). Analyses showed that higher P intake was associated with higher $\mathrm{Ca}$ intake, and that dietary Ca:P ratios (0.51-0.62, with a mean of 0.60 for adults) were adequate in all age/gender groups. High intake of P was positively associated with BMC in female teenagers (Q4 vs. Q1: BMC, $30.9 \pm 1.1$ vs. $29.0 \pm 0.5 \mathrm{~g}, \mathrm{P}=0.001$ ). It was also positively associated with $B M C$ and $B M D$ as well as reduced risk of osteoporosis in adults $>20$ years of age (Q4 vs. Q1: OR of osteoporosis, 0.55 ; $95 \%$ confidence interval [Cl], 0.39- 0.79; $\mathrm{P}=0.001 ; \mathrm{BMC}, 37.5 \pm 0.4$ vs. $36.70 \pm 0.3 \mathrm{~g}, \mathrm{P}<0.01 ; \mathrm{BMD}, 0.986 \pm 0.004$ vs. $\left.0.966 \pm 0.005 \mathrm{~g} / \mathrm{cm}^{2}, \mathrm{P}<0.05\right)$. The data suggest that high intake of $\mathrm{P}$ has no adverse effect on bone metabolism in populations with adequate $\mathrm{Ca}$ intake, and that it is also associated with positive bone parameters in some age/gender groups.
\end{abstract}

Keywords: Phosphorus intake, Bone mineral content, Bone mineral density, Osteoporosis

\section{Introduction}

Maintenance of healthy bones throughout the lifespan is physiologically beneficial. Greater bone formation and/or less bone resorption lead to an increase in BMC and/or $\mathrm{BMD}$. Assessment of BMD is used to define osteoporosis, a systemic skeletal disease characterized by low BMD and abnormal bone micro-architecture. These deficits lead to fragility and increased susceptibility to fractures [1]. BMC and BMD are considered good biomarkers for bone health [2]. The main factors that affect maximum bone mass and strength are genetic predisposition and dietary habits [3]. Dietary interventions (e.g., Ca supplementation) increase $\mathrm{BMD}$ or limit loss of it in older adults and postmenopausal females, reducing the risk of osteoporotic fractures.

The effects of high $\mathrm{P}$ intake on bone parameters have been controversial. Many studies report that high P intake is harmful to bone health in subjects whose dietary Ca:P ratio is extremely low [4-10]. However, there is strong evidence that high $\mathrm{P}$ intake has no negative

\footnotetext{
* Correspondence: susanscho1@yahoo.com

${ }^{2}$ NutraSource, 6309 Morning Dew Ct., Clarksville, MD 21029, USA

Full list of author information is available at the end of the article
}

impact on Ca balance in normal subjects with appropriate dietary $\mathrm{Ca}$ and $\mathrm{P}$ intakes [11-15].

We hypothesized that in those with adequate Ca intake, the adverse effects of higher $\mathrm{P}$ intake on bone metabolism would be negligible. Therefore, we examined the association between $\mathrm{P}$ intake and bone health parameters in a cross-sectional cohort of the 2005-2010 NHANES.

\section{Methods}

All analyses were completed using Statistical Analysis Software (SAS) 9.2 and SUDAAN version 11 and used sampling weights, primary sampling units and strata information provided by NHANES. Day 1 dietary, exam, lab and questionnaire data were used for NHANES 2005-2010 with exclusions for age $<13$, incomplete or unreliable data and pregnant or lactating females. All analyses were done for males, females and genders combined. The age groups for the analyses were 13-19, 20-49, 50-99 and 20-99. The Osteoporosis questionnaire (OSQ) files were used to indicate presence of osteoporosis. The Prescription Medication (RXQ) files were used to identify subjects taking osteoporosis or estrogen medications. The Dual Energy 
X-ray Absorptiometry-Femur files were used for BMD $\left(\mathrm{g} / \mathrm{cm}^{2}\right)$ and BMC $(\mathrm{g})$ measurements.

Phosphorus quartiles were calculated by age group and gender using SUDAAN proc descript. Means by phosphorus intake quartile for nutrient intakes, questionnaire responses and lab results were calculated using SUDAAN proc regress with separate regressions within age groups and gender using indicator variables for the P intake quartiles.

Least Square Means (LSM) and standard errors (SE) were calculated for total femur BMC and BMD across the quartiles of phosphorus intake using SUDAAN proc regress by age groups and gender. P-value statistics were calculated for quartile trend on quartile numbers on phosphorus intake in grams. The LSM analysis was done after adjustments for age, gender, energy, body mass index (BMI), protein intake, diary intake, calcium intake, vitamin D intake and flags for supplemental intake of calcium, vitamin D, and multi-vitamins. Flags were also included for taking osteoporosis medication and, for females, taking estrogen medication.

Odds ratios were calculated for the indication of osteoporosis across the quartiles of phosphorus intake using SUDAAN proc rlogist by age groups and genders. The same trend statistics were calculated as in the LSM analysis. The covariate sets used were as used in the LSM analysis except the flag for osteoporosis was not included. The 13-19 age group was not used in this logistic analysis since the NHANES questionnaire for osteoporosis does not include this age group. Two flags were used as indicators of osteoporosis. These were based on the prescription medication rx_drug files and the osteoporosis questionnaire osq files. Osteoporosis was determined as indicated if one or more of 2 conditions were satisfied: (1) Answer yes to question "Has a doctor ever told you that you had osteoporosis, sometimes called thin or brittle bones?" and (2) Answer yes to question "Has a doctor ever told you that you had broken or fractured your hip?".

\section{Results and discussion}

The importance of $\mathrm{Ca}$ and $\mathrm{P}$ in maintaining bone health is well established [3]. Based on Ca retention from balance studies, factorial estimates of requirements, and data on changes in BMD and BMC, the Institute of Medicine (IOM) [3] set an adequate intake (AI) of calcium at $1,200 \mathrm{mg} /$ day for adults $>50$ years of age and a Tolerable Upper Intake Level (UL) at 2,500 mg/day. The Recommended Dietary Allowances (RDA) and UL for P are 700 and 4,000 $\mathrm{mg} /$ day for adults, respectively, and 1,250 and $4,000 \mathrm{mg} /$ day in teenagers [3].

\section{Characteristics of the NHANES population and intakes of $\mathrm{P}$ and $\mathrm{Ca}$}

Table 1 shows characteristics of the NHANES population. Compared with teenagers and adults aged 20-49 years, those aged $\geq 50$ years took in less dietary Ca and P, soft drinks, and dairy foods. They also used more supplemental $\mathrm{Ca}$ and multivitamins. The LSM of various ethnic groups were compared for intakes of $\mathrm{Ca}$ and $\mathrm{P}$. Whites had the highest intakes of both $\mathrm{Ca}$ and P; Blacks had the lowest (Whites vs. Blacks: Ca, 1,013 vs. $799 \mathrm{mg} /$ day, $\mathrm{P}<0.01$; $\mathrm{P}$, 1,409 vs. $1,188 \mathrm{mg} /$ day, $\mathrm{P}<0.01$; data not shown in tables).

Table 2 shows that high $\mathrm{P}$ consumers $(\mathrm{Q} 4)$ had $\mathrm{P}$ intake two to three times higher than the RDA. It was approximately $2.4 \mathrm{~g} /$ day, well below the UL of $4 \mathrm{~g} /$ day. None of the high $\mathrm{P}$ intake groups had $\mathrm{P}$ intake over the UL of $4.0 \mathrm{~g} /$ day. Data also showed that higher P intake was associated with higher $\mathrm{Ca}$ intake.

Dietary intake of Ca was approximately $1,000 \mathrm{mg} /$ day, with that among high $\mathrm{P}$ consumers 1.5-fold higher than the recommended RDA (1,620-1,890 mg/day). Ca intake was well below the UL of $2,500 \mathrm{mg} /$ day [3]. None of the high P intake groups had Ca intake over the UL of $2.5 \mathrm{~g} /$ day. According to nationwide food consumption surveys, milk was the leading source of $\mathrm{P}$ as well as $\mathrm{Ca}$ in all age groups [16]. In all age/gender groups, NHANES data indicated that the dietary Ca:P molar ratio was adequate, with a range of 0.51-0.62. Compared with females, males had higher intakes of Ca and P (Table 2).

\section{Markers of bone metabolism and OR of osteoporosis}

A multivariate model that included adjustments for intakes of calcium, protein, and vitamin $\mathrm{D}$ showed that

Table 1 Demographics of NHANES subjects

\begin{tabular}{|c|c|c|c|c|}
\hline Age group & $13-19$ years & $20-49$ years & $50-99$ years & $20-99$ years \\
\hline Phosphorus (mg) & $1382 \pm 192^{a}$ & $1443 \pm 14^{b}$ & $1259 \pm 20^{c}$ & $1368 \pm 11$ \\
\hline Calcium (mg) & $1070 \pm 19^{a}$ & $1020 \pm 13^{b}$ & $888 \pm 17^{c}$ & $966 \pm 10$ \\
\hline Energy (kcal) & $2255 \pm 27^{a}$ & $2309 \pm 21^{b}$ & $1896 \pm 28^{c}$ & $2147 \pm 16$ \\
\hline Total Dairy (oz equivalent) & $2.1 \pm 0.05^{\mathrm{a}}$ & $1.8 \pm 0.04^{b}$ & $1.4 \pm 0.04^{c}$ & $1.6 \pm 0.0$ \\
\hline Soft Drinks (oz) & $14.9 \pm 0.7^{a}$ & $17.2 \pm 0.6^{b}$ & $9.6 \pm 0.43^{c}$ & $14.0 \pm 0.4$ \\
\hline Supplemental Calcium (\%) & $20.8 \pm 1.2^{\mathrm{a}}$ & $40.2 \pm 0.8^{b}$ & $56.6 \pm 1.6^{c}$ & $46.9 \pm 0.7$ \\
\hline Multivitamin use (\%) & $18.8 \pm 1.3^{\mathrm{a}}$ & $32.0 \pm 0.9^{b}$ & $51.1 \pm 1.7^{c}$ & $38.8 \pm 0.8$ \\
\hline Osteoporosis medication (\%) & NA & $0.2 \pm 0.06^{\mathrm{a}}$ & $5.3 \pm 0.58^{b}$ & $2.0 \pm 0.15$ \\
\hline
\end{tabular}

$\mathrm{NA}=$ not applicable. ${ }^{\mathrm{a}, \mathrm{b}, \mathrm{c}}$ Different superscripts indicate statistically significantly different values from other age groups. 
Table 2 Phosphorus and Calcium intakes, mg/day

\begin{tabular}{|c|c|c|c|c|c|}
\hline Gender & LSM & Q1 & Q2 & Q3 & Q4 \\
\hline \multicolumn{6}{|c|}{ Phosphorus intakes, mg/day } \\
\hline 13-19 yr, All & $1382 \pm 14$ & $597 \pm 7$ & $1037 \pm 5$ & $1471 \pm 8$ & $2421 \pm 39$ \\
\hline 13-19 yr, Male & $1624 \pm 23^{\mathrm{a}}$ & $599 \pm 15$ & $1055 \pm 8$ & $1476 \pm 10$ & $2484 \pm 49$ \\
\hline 13-19 yr, Female & $1138 \pm 10^{b}$ & $596 \pm 8$ & $1023 \pm 6$ & $1465 \pm 10$ & $2247 \pm 62$ \\
\hline -49 yr, All & $1443 \pm 6$ & $684 \pm 6$ & $1121 \pm 3$ & $1541 \pm 4$ & $2426 \pm 21$ \\
\hline 20-49 yr, Male & $1701 \pm 10^{a}$ & $718 \pm 9$ & $1126 \pm 6$ & $1548 \pm 5$ & $2487 \pm 26$ \\
\hline 0-49 yr, Female & $1179 \pm 4^{b}$ & $671 \pm 8$ & $1118 \pm 5$ & $1531 \pm 6$ & $2231 \pm 32$ \\
\hline 50-99 yr, All & $1377 \pm 4$ & $701 \pm 6$ & $1091 \pm 3$ & $1451 \pm 4$ & $2260 \pm 17$ \\
\hline 50-99 yr, Male & $1592 \pm 8^{\mathrm{a}}$ & $698 \pm 7$ & $1087 \pm 5$ & $1462 \pm 5$ & $2315 \pm 23$ \\
\hline -99 yr, Female & $1172 \pm 4^{b}$ & $703 \pm 7$ & $1094 \pm 4$ & $1438 \pm 5$ & $2117 \pm 22$ \\
\hline 20-99 yr, All & $1368 \pm 4$ & $667 \pm 4$ & $1071 \pm 2$ & $1450 \pm 3$ & $2283 \pm 14$ \\
\hline 20-99 yr, Male & $1602 \pm 7^{\mathrm{a}}$ & $693 \pm 6$ & $1075 \pm 3$ & $1457 \pm 4$ & $2348 \pm 19$ \\
\hline 20-99 yr, Female & $1145 \pm 4^{b}$ & $657 \pm 5$ & $1068 \pm 2$ & $1441 \pm 5$ & $2110 \pm 21$ \\
\hline \multicolumn{6}{|c|}{ Calcium intakes according to phosphorus intake quartiles, mg/day } \\
\hline 3-19 yr, All & $1070 \pm 14$ & $470 \pm 11$ & $768 \pm 15$ & $1144 \pm 20$ & $1896 \pm 45$ \\
\hline 13-19 yr, Male & $1243 \pm 23^{a}$ & $464 \pm 15$ & $797 \pm 23$ & $1114 \pm 28$ & $1918 \pm 53$ \\
\hline 3-19 yr, Female & $894.8 \pm 16^{\mathrm{b}}$ & $472 \pm 14$ & $747 \pm 17$ & $1182 \pm 24$ & $1833 \pm 83$ \\
\hline 20-49 yr, All & $1020.5 \pm 7$ & $496 \pm 9$ & $791 \pm 12$ & $1061 \pm 10$ & $1732 \pm 25$ \\
\hline-49 yr, Male & $1157 \pm 11^{\mathrm{a}}$ & $477 \pm 14$ & $746 \pm 19$ & $1004 \pm 13$ & $1742 \pm 30$ \\
\hline 20-49 yr, Female & $880 \pm 8^{b}$ & $503 \pm 10$ & $824 \pm 14$ & $1137 \pm 16$ & $1700 \pm 41$ \\
\hline 50-99 yr, All & $895 \pm 6$ & $462 \pm 9$ & $710 \pm 8$ & $946 \pm 13$ & $1460 \pm 18$ \\
\hline 0-99 yr, Male & $985 \pm 8^{\mathrm{a}}$ & $437 \pm 11$ & $678 \pm 11$ & $888 \pm 11$ & $1461 \pm 22$ \\
\hline 50-99 yr, Female & $817 \pm 8^{b}$ & $472 \pm 10$ & $730 \pm 11$ & $1001 \pm 22$ & $1460 \pm 29$ \\
\hline 20-99 yr, All & $966 \pm 5$ & $481 \pm 5$ & $750 \pm 6$ & $1010 \pm 8$ & $1622 \pm 18$ \\
\hline 20-99 yr, Male & $1086 \pm 8^{a}$ & $458 \pm 7$ & $713 \pm 13$ & $9529 \pm 9$ & $1631 \pm 21$ \\
\hline 0-99 yr, Female & $852 \pm 7^{b}$ & $490 \pm 6$ & $775 \pm 8$ & $1079 \pm 14$ & $1597 \pm 27$ \\
\hline \multicolumn{6}{|c|}{ Dietary Ca:P intake molar ratio } \\
\hline 13-19 yr, All & 0.60 & 0.62 & 0.57 & 0.60 & 0.60 \\
\hline 3-19 yr, Male & 0.59 & 0.59 & 0 & 0. & 0.58 \\
\hline 13-19 yr, Female & 0.60 & 0.64 & 0.57 & 0.59 & 0.62 \\
\hline $0-49$ yr, All & 0.55 & 0.57 & 0.54 & 0.53 & 0.55 \\
\hline 20-49 yr, Male & 0.52 & 0.51 & 0.52 & 0.50 & 0.54 \\
\hline 20-49 yr, Female & 0.58 & 0.61 & 0.56 & 0.56 & 0.59 \\
\hline -99 yr, All & 0.55 & 0.56 & 0.54 & 0.54 & 0.54 \\
\hline 50-99 yr, Male & 0.52 & 0.51 & 0.51 & 0.51 & 0.54 \\
\hline 50-99 yr, Female & 0.57 & 0.58 & 0.57 & 0.57 & 0.57 \\
\hline 20-99 yr, All & 0.55 & 0.57 & 0.54 & 0.54 & 0.54 \\
\hline 20-99 yr, Male & 0.52 & 0.51 & 0.51 & 0.51 & 0.54 \\
\hline 20-99 yr, Female & 0.58 & 0.60 & 0.57 & 0.57 & 0.58 \\
\hline
\end{tabular}

a,b Different superscripts indicate statistically significantly different values between males and females.

high $\mathrm{P}$ intake combined with adequate $\mathrm{Ca}$ was associated with improved bone health. This was indicated by improved $\mathrm{BMC}, \mathrm{BMD}$, and/or reduced $\mathrm{OR}$ of osteoporosis in some age/gender groups. In all age/gender groups with higher Ca intake, increased $\mathrm{P}$ had no adverse effects on bone health parameters. The dietary $\mathrm{Ca}: \mathrm{P}$ ratio was within the safe intake range. $\mathrm{P}$ intake was not associated with serum concentrations of vitamin D or $\mathrm{P}$ (data not shown). Compared with females, males had higher values for BMC and BMD (Tables 3 and 4).

Table 3 shows quartiles of the LSM of total femur $\mathrm{BMC}$ and a linear trend in $\mathrm{P}(\mathrm{mg})$. Data show a positive association between $\mathrm{P}$ intake and increasing $\mathrm{BMC}$ among those aged $20-99$ years $(3.4-4.2 \% ; \mathrm{P}<0.05)$, in those aged $20-49$ years $(4.6 \% ; \mathrm{P}<0.01)$, and females aged $13-19$ years $(6.6 \%$; $<<0.01)$. Increases in $P$ intake were associated with significant gains in total femur BMC. In males aged 20-49 years, there was an increasing trend of $\mathrm{BMC}$ in response to high $\mathrm{P}$ intake (4.0\%; $\mathrm{P}=0.10$ ).

Of those aged 20-99 years, assessment by ethnic group attenuated statistical power for differentiation. Increased $\mathrm{P}$ intake was associated with higher BMC only in Whites (Q4 vs. Q1: 37.5 vs. 36.0; $\mathrm{P}<0.01$ ), with an increasing trend of $\mathrm{BMC}(\mathrm{Q} 4$ vs. Q1: 38.8 vs. 37.7; $\mathrm{P}=0.09)$ in Black participants. A comparison of LSMs showed that whites and Hispanics had significantly lower BMC than blacks, but it was higher than the level in other ethnic groups (including Asians).

Table 4 reports total femur BMD in each quartile. High P intake in those aged 20-99 years was associated with a $2.1 \%$ increase in total femur BMD. Similar increases were observed in all adults and females aged 2049 years. In these groups, the highest quartile of $\mathrm{P}$ intake produced 2.0-2.4\% greater BMD compared with the lowest quartile. In teenagers, $\mathrm{P}$ intake was not associated with BMD.

When those aged 20-99 years were compared by ethnicity, increased P intakes were associated with higher BMD only in Whites (Q4 vs. Q1: 0.976 vs. 0.946; $\mathrm{P}<0.01$ ). Whites and Hispanics had significantly lower BMD than blacks, but levels were higher than in other ethnic groups (including Asians). In adults aged $\geq 20$ years, $P$ intake was associated with improved bone health as indicated by increased BMD. In contrast, the Framingham Osteoporosis Study [17] found that total P intake was not related to BMD in 1,413 females and 1,125 males whose dietary Ca: $\mathrm{P}$ ratios ranged from 0.60 to 0.62 .

Table 5 shows the OR of osteoporosis by quartile. Males and females aged 20-99 years with the highest $\mathrm{P}$ intake had a $45 \%$ lower risk of osteoporosis (OR = 0.55; 95\% CI, 0.39-0.79; $\mathrm{P}<0.01)$ compared with those who consumed the least P. When NHNANES subjects were divided into two age groups, 20-49 and 50-99, statistical power was attenuated. High P intake was associated with a decreasing trend for osteoporosis. Risk reduction was $54 \%(\mathrm{P}=0.10)$ in those aged $20-49$ years. In those aged $50-99$ years, it was $29 \%(\mathrm{P}=0.06)$. 
Table 3 Femur Bone mineral content (BMC) according to $P$ intake quartiles

\begin{tabular}{|c|c|c|c|c|c|c|}
\hline Age, gender & LSM & Q1 & Q2 & Q3 & Q4 & $\mathrm{P}$ trend \\
\hline 13-19 yr, All & $35.1 \pm 0.2$ & $34.6 \pm 0.5$ & $34.9 \pm 0.3$ & $35.5 \pm 0.3$ & $35.2 \pm 0.5$ & NS \\
\hline 13-19 yr, Male & $40.1 \pm 0.3^{\mathrm{a}}$ & $39.5 \pm 0.8$ & $40.0 \pm 0.7$ & $41.1 \pm 0.5$ & $39.6 \pm 0.6$ & NS \\
\hline 13-19 yr, Female & $29.6 \pm 0.2^{b}$ & $29.0 \pm 0.5$ & $29.9 \pm 0.3$ & $29.5 \pm 0.5$ & $30.9 \pm 1.1$ & $<0.01$ \\
\hline 20-49 yr, All & $37.4 \pm 0.1$ & $36.5 \pm 0.3$ & $37.3 \pm 0.2$ & $37.5 \pm 0.3$ & $38.2 \pm 0.3$ & $<0.01$ \\
\hline 20-49 yr, Male & $43.8 \pm 0.2^{a}$ & $42.9 \pm 0.7$ & $43.4 \pm 0.4$ & $43.4 \pm 0.4$ & $44.6 \pm 0.4$ & 0.1010 \\
\hline 20-49 yr, Female & $30.6 \pm 0.1^{b}$ & $29.9 \pm 0.4$ & $30.9 \pm 0.2$ & $31.4 \pm 0.3$ & $31.0 \pm 0.6$ & $<0.05$ \\
\hline 50-99 yr, All & $35.8 \pm 0.1$ & $35.5 \pm 0.4$ & $35.6 \pm 0.2$ & $36.0 \pm 0.2$ & $35.9 \pm 0.4$ & NS \\
\hline 50-99 yr, Male & $43.6 \pm 0.3^{a}$ & $43.1 \pm 1.0$ & $43.2 \pm 0.8$ & $44.0 \pm 0.6$ & $43.7 \pm 0.7$ & NS \\
\hline 50-99 yr, Female & $28.6 \pm 0.1^{b}$ & $28.6 \pm 0.4$ & $28.6 \pm 0.2$ & $28.5 \pm 0.2$ & $28.4 \pm 0.5$ & NS \\
\hline 20-99 yr, All & $36.7 \pm 0.1$ & $36.0 \pm 0.3$ & $36.4 \pm 0.2$ & $36.8 \pm 0.2$ & $37.5 \pm 0.4$ & $<0.01$ \\
\hline 20-99 yr, Male & $43.7 \pm 0.1^{\mathrm{a}}$ & $42.9 \pm 0.5$ & $43.2 \pm 0.3$ & $43.4 \pm 0.2$ & $44.5 \pm 0.3$ & $<0.05$ \\
\hline 20-99 yr, Female & $29.7 \pm 0.1^{b}$ & $29.3 \pm 0.3$ & $29.7 \pm 0.2$ & $30.1 \pm 0.2$ & $30.3 \pm 0.4$ & $<0.05$ \\
\hline \multicolumn{7}{|c|}{ Comparison of ethnicity; 20-99 yr, All (males and females combined) } \\
\hline Hispanic & $35.9 \pm 0.16^{t}$ & $35.7 \pm 0.32$ & $36.1 \pm 0.31$ & $35.8 \pm 0.20$ & $36.0 \pm 0.32$ & NS \\
\hline White & $36.9 \pm 0.14^{x}$ & $36.0 \pm 0.28$ & $36.5 \pm 0.23$ & $37.0 \pm 0.21$ & $37.5 \pm 0.25$ & $<0.01$ \\
\hline Black & $38.2 \pm 0.21^{y}$ & $37.7 \pm 0.48$ & $37.8 \pm 0.28$ & $38.8 \pm 0.35$ & $38.8 \pm 0.60$ & 0.09 \\
\hline Other & $33.5 \pm 0.33^{\mathrm{t}, \mathrm{z}}$ & $32.9 \pm 0.67$ & $33.4 \pm 0.56$ & $32.8 \pm 0.36$ & $35.3 \pm 1.01$ & NS \\
\hline
\end{tabular}

Femur BMCs were calculated for each quartile after adjustments for age, gender, kcal, BMI, intakes of calcium, protein, total dairy foods, and vitamin D, and flags to denote supplemental calcium, supplemental vitamin D, supplemental multi vitamin/mineral and a flag to denote prescription medication for osteoporosis and estrogen medication (females only).

${ }^{\mathrm{a}, \mathrm{b}}$ Different superscripts indicate statistically significantly different LSM values between males and females.

$t, x, y, z$ Different superscripts indicate statistically significantly different LSM values in 4 ethnic groups.

Table 4 Femur Bone mineral density (BMD) according to $P$ intake quartiles

\begin{tabular}{|c|c|c|c|c|c|c|}
\hline Gender & LSM & Q1 & Q2 & Q3 & Q4 & $P$ trend \\
\hline 13-19 yr, All & $1.000 \pm 0.003$ & $0.990 \pm 0.01$ & $0.999 \pm 0.006$ & $1.003 \pm 0.005$ & $1.009 \pm 0.008$ & NS \\
\hline 13-19 yr, Male & $1.038 \pm 0.005^{a}$ & $1.027 \pm 0.02$ & $1.039 \pm 0.013$ & $1.048 \pm 0.008$ & $1.035 \pm 0.010$ & NS \\
\hline 13-19 yr, Female & $0.959 \pm 0.004^{b}$ & $0.945 \pm 0.011$ & $0.963 \pm 0.006$ & $0.956 \pm 0.011$ & $0.993 \pm 0.021$ & NS \\
\hline 20-49 yr, All & $1.014 \pm 0.002$ & $0.999 \pm 0.006$ & $1.016 \pm 0.004$ & $1.017 \pm 0.005$ & $1.023 \pm 0.006$ & $<0.05$ \\
\hline 20-49 yr, Male & $1.062 \pm 0.004^{\mathrm{a}}$ & $1.058 \pm 0.012$ & $1.058 \pm 0.006$ & $1.056 \pm 0.006$ & $1.069 \pm 0.008$ & NS \\
\hline 20-49 yr, Female & $0.964 \pm 0.003^{b}$ & $0.948 \pm 0.007$ & $0.970 \pm 0.005$ & $0.977 \pm 0.007$ & $0.971 \pm 0.010$ & $<0.05$ \\
\hline 50-99 yr, All & $0.924 \pm 0.002$ & $0.919 \pm 0.006$ & $0.923 \pm 0.005$ & $0.927 \pm 0.004$ & $0.926 \pm 0.007$ & NS \\
\hline 50-99 yr, Male & $1.001 \pm 0.004^{\mathrm{a}}$ & $0.996 \pm 0.009$ & $1.005 \pm 0.009$ & $1.004 \pm 0.006$ & $0.998 \pm 0.008$ & NS \\
\hline 50-99 yr, Female & $0.853 \pm 0.003^{b}$ & $0.858 \pm 0.008$ & $0.849 \pm 0.006$ & $0.852 \pm 0.006$ & $0.852 \pm 0.011$ & NS \\
\hline 20-99 yr, All & $0.976 \pm 0.002$ & $0.966 \pm 0.005$ & $0.973 \pm 0.004$ & $0.977 \pm 0.004$ & $0.986 \pm 0.004$ & $<0.05$ \\
\hline 20-99 yr, Male & $1.037 \pm 0.003^{\mathrm{a}}$ & $1.034 \pm 0.008$ & $1.036 \pm 0.005$ & $1.029 \pm 0.004$ & $1.044 \pm 0.005$ & NS \\
\hline 20-99 yr, Female & $0.915 \pm 0.002^{b}$ & $0.909 \pm 0.005$ & $0.912 \pm 0.004$ & $0.922 \pm 0.005$ & $0.924 \pm 0.008$ & NS \\
\hline \multicolumn{7}{|c|}{ Comparison of ethnicity; 20-99 yr, All (males and females combined) } \\
\hline Hispanic & $0.997 \pm 0.003^{t, z}$ & $0.986 \pm 0.007$ & $1.002 \pm 0.007$ & $1.002 \pm 0.004$ & $0.997 \pm 0.007$ & NS \\
\hline White & $0.964 \pm 0.002^{\mathrm{t}, \mathrm{x}}$ & $0.946 \pm 0.006$ & $0.958 \pm 0.004$ & $0.967 \pm 0.004$ & $0.976 \pm 0.004$ & $<0.01$ \\
\hline Black & $1.048 \pm 0.004^{y}$ & $1.041 \pm 0.008$ & $1.043 \pm 0.005$ & $1.054 \pm 0.007$ & $0.968 \pm 0.010$ & NS \\
\hline Other & $0.944 \pm 0.005^{z}$ & $0.932 \pm 0.016$ & $0.955 \pm 0.012$ & $0.926 \pm 0.010$ & $0.968 \pm 0.022$ & NS \\
\hline
\end{tabular}

Femur BMDs were calculated for each quartile after adjustments for age, gender, kcal, BMI, intakes of calcium, protein, total dairy foods, and vitamin D, and flags to denote supplemental calcium, supplemental vitamin D, supplemental multi vitamin/mineral and a flag to denote prescription medication for osteoporosis and estrogen medication (females only).

${ }^{a, b}$ Different superscripts indicate statistically significantly different LSM values between males and females or among different ethnic groups.

$t, x, y, z$ Different superscripts indicate statistically significantly different LSM values in 4 ethnic groups. 
Table 5 Odd ratio of osteoporosis according to $P$ intake quartiles

\begin{tabular}{|c|c|c|c|c|c|}
\hline \multirow[b]{2}{*}{ Gender } & \multicolumn{5}{|c|}{ Odd ratio } \\
\hline & $\overline{\mathrm{Q} 1}$ & Q2 & Q3 & Q4 & $P$ trend $^{\mathrm{a}}$ \\
\hline 20-49 yr, All & 1.00 & $0.39(0.19,0.78)$ & $0.54(0.26,1.12)$ & $0.46(0.16,1.27)$ & 0.10 \\
\hline 20-49 yr, Male & 1.00 & $0.36(0.11,1.20)$ & $0.51(0.16,1.60)$ & $0.60(0.15,2.38)$ & NS \\
\hline 20-49 yr, Female & 1.00 & $0.37(0.18,1.00)$ & $0.73(0.29,1.39)$ & $0.27(0.06,1.33)$ & NS \\
\hline 50-99 yr, All & 1.00 & $0.84(0.63,1.11)$ & $0.77(0.55,1.08)$ & $0.71(0.48,1.04)$ & 0.06 \\
\hline 50-99 yr, Male & 1.00 & $0.45(0.23,0.89)$ & $0.55(0.26,1.15)$ & $0.52(0.17,1.53)$ & NS \\
\hline 50-99 yr, Female & 1.00 & $1.01(0.71,1.44)$ & $0.98(0.61,1.59)$ & $1.05(0.52,2.09)$ & NS \\
\hline 20-99 yr, All & 1.00 & $0.76(0.59,0.97)$ & $0.68(0.51,0.91)$ & $0.55(0.39,0.79)$ & $<0.001$ \\
\hline 20-99 yr, Male & 1.00 & $0.49(0.30,0.80)$ & $0.56(0.32,0.97)$ & $0.46(0.22,1.00)$ & 0.08 \\
\hline 20-99 yr, Female & 1.00 & $0.85(0.58,1.24)$ & $0.78(0.44,1.36)$ & $0.65(0.31,1.38)$ & NS \\
\hline \multicolumn{6}{|c|}{ Comparison of ethnicity; 20-99 yr, All (males and females combined) } \\
\hline Hispanic & 1.00 & $0.56(0.61,0.96)$ & $0.61(0.32,1.14)$ & $0.37(0.15,0.91)$ & 0.07 \\
\hline White & 1.00 & $0.82(0.63,1.07)$ & $0.64(0.39,1.05)$ & $0.67(0.34,1.32)$ & 0.11 \\
\hline Black & 1.00 & $0.69(0.41,1.18)$ & $1.17(0.63,2.19)$ & $1.31(0.49,3.54)$ & NS \\
\hline Other & 1.00 & $0.40(0.11,1.50)$ & $0.27(0.07,0.97)$ & $0.13(0.01,1.53)$ & 0.05 \\
\hline
\end{tabular}

Osteoporosis was determined as indicated if one or more of 2 conditions were satisfied: (1) Answer yes to question "Has a doctor ever told you that you had osteoporosis, sometimes called thin or brittle bones?" and (2) Answer yes to question "Has a doctor ever told you that you had broken or fractured your hip?". ORs for osteoporosis were calculated for each quartile after adjustments for age, gender, kcal, BMl, intakes of calcium, protein, total dairy foods, and vitamin $D$, and flags to denote supplemental calcium, supplemental vitamin D, supplemental multi vitamin/mineral and prescription medication for osteoporosis and estrogen medication (females only).

${ }^{a} \mathrm{P}$ trend values indicate statistically significantly different trends in 4 quartiles.

Increased P intake was associated with a lower OR of osteoporosis in various ethnic groups, with a decreasing trend in whites $(\mathrm{OR}=0.67 ; 95 \% \mathrm{CI}, 0.34,1.32 ; \mathrm{P}=0.11)$ and Hispanics $(\mathrm{OR}=0.37 ; 95 \% \mathrm{CI}, 0.15,0.91 ; \mathrm{P}=0.07)$. Other ethnic groups had a $87 \%$ lower risk of osteoporosis $(\mathrm{P}<0.05)$.

Several studies show that high $\mathrm{P}$ intake does not have a negative impact on Ca balance. Spencer et al. [11] found that an increase in P intake from $800 \mathrm{mg} /$ day (the RDA) to $2,000 \mathrm{mg} /$ day in adult males failed to affect Ca balance regardless of intake, which ranged from 200 to 2,000 mg/ day. Similarly, Heany and colleagues [12-15] reported that intake of dietary $\mathrm{P}$ was inversely associated with both urinary $\mathrm{Ca}$ excretion and intestinal $\mathrm{Ca}$ absorption, implying a direct association with fecal $\mathrm{Ca}$ excretion. Changes in $\mathrm{P}$ intake by healthy adults affect $\mathrm{Ca}$ metabolism (i.e., decreased intestinal $\mathrm{Ca}$ absorption and decreased renal excretion of $\mathrm{Ca}$ ). However, these outcomes most likely cancel each other out, leaving Ca balance unaffected.

In several studies, a high ratio of $\mathrm{P}: \mathrm{Ca}$ (approximately 3-4:1) in conjunction with low Ca intake led to adverse effects on bone health. Many of these reports had small numbers of subjects or were of short duration. For example, a cross-sectional study of 38 young females found a negative association between $\mathrm{P}$ intake and radial bone measurements [9]. Meaningful data interpretation was unlikely with such a small sample. A cross-sectional observational study of 147 healthy females aged 31-43 years by Kemi et al. [6,7] reported that with adequate $\mathrm{Ca}$ intake, the lowest quartile (Ca:P ratio of $<0.51)$ had higher mean serum parathyroid hormone $(\mathrm{S}-\mathrm{PTH})$ concentration $(\mathrm{P}=$ 0.021) and mean urinary $\mathrm{Ca}(\mathrm{U}-\mathrm{Ca})$ excretion $(\mathrm{P}=0.051)$ than all other quartiles. It should be noted that the number of subjects in the study was too small for an observational study to make a reliable conclusion. Randomized clinical trials have shown that the combination of high P (1,500-1,700 mg) coupled with low Ca intake (375$400 \mathrm{mg}$ ) increased serum PTH concentration in healthy young females $[4,5,8]$. Portale et al. [10] found that a high intake of $\mathrm{P}$ decreased the serum calcitriol concentration in healthy males. It also acutely inhibited bone formation [4].

Kemi et al. [18] reported that when P intake was above current recommendations, increased $\mathrm{Ca}$ intake was beneficial for bone, as indicated by decreased S-PTH concentration and bone resorption; not even high $\mathrm{Ca}$ intake had effect on bone formation when $\mathrm{P}$ intake was excessive. In this study, 12 healthy female subjects aged 21-40 years attended three 24-h study sessions. They were randomized according to a Ca dose of 0 (control day), 600, or 1,200 mg, and each subject served as her own control. The meals on each study day provided 1,850 mg P and $480 \mathrm{mg}$ Ca. Rising Ca doses were accompanied by decreasing concentrations of serum PTH $(\mathrm{P}<0.001)$ and increasing serum ionized Ca concentrations $(\mathrm{P}<0.001)$, with no significant differences in the bone formation marker and serum bonespecific alkaline phosphatase. It is worth noting that the study duration of this study was too short to see a meaningful impact on bone health parameters. 
In the U.S., mineral intake is generally adequate. The IOM [3] stated that in balance studies, Ca:P molar ratios of 0.08-2.4:1 (a 30 fold range) had no effect on either $\mathrm{Ca}$ balance or absorption. The IOM also found that the intake ratio alone failed to take into account physiological adaptive responses, such as bioavailability and renal output. For example, in term-born infants during the first year of life, a higher $\mathrm{Ca}$ content in soy-based formulas reduced $\mathrm{P}$ absorption, but retention was similar due to offsetting changes in renal $\mathrm{P}$ output. Thus, the IOM concluded that there is little evidence for relating the two nutrients.

Adequate dietary $\mathrm{P}$ is essential for building bone since bone mineral is predominantly calcium phosphate [3]. Milk is the leading source of dietary P in the American diet, but a synergistic effect of other nutrients in milk (e.g., calcium, protein, potassium, magnesium) may improve absorption of dairy $\mathrm{Ca}$ [14]. Although Ca supplements reduce its absorption compared to dairy milk [14], those that contain P (e.g., tricalcium phosphate, dicalcium phosphate) maintain strong bones and reduce the risk of osteoporosis [15]. Our data show that high P intake is associated with high $\mathrm{Ca}$ intake as well as improved bone health in some age/gender groups.

Other findings demonstrate beneficial effects of $\mathrm{Ca}$ phosphate supplementation. Anabolic agents for treating osteoporosis require positive $\mathrm{P}$ balances of up to $90 \mathrm{mg} /$ day [19]. A Ca phosphate supplement may be preferable to carbonate or citrate salts because it preserves food $\mathrm{P}$ [15]. Ditscheid et al. [20] reported that pentacalcium hydroxy-triphosphate $\left(\mathrm{Ca}_{5}\left(\mathrm{PO}_{4}\right)_{3} \mathrm{OH}\right)$ supplementation provided additional daily intake of $1060 \mathrm{mg} \mathrm{Ca}$ and $490 \mathrm{mg}$ P. These amounts significantly lowered serum total and LDL cholesterol concentrations by $6.5 \%$ and $3.6 \%$, respectively, after 4 weeks of supplementation.

This study has several shortcomings. First, its crosssectional design precludes the assignment of cause and effect. A prospective, longitudinal study may be more appropriate for a population study. Second, lack of PTH data in the NHANES dataset did not allow analysis of the impact of high P intake on blood PTH concentrations. Additional studies with parameters beyond bone health should be considered before increasing P consumption.

\section{Conclusion}

Our cross-sectional analyses of NHANES data show that high $\mathrm{P}$ intake is associated with a $4.2 \%$ improvement in BMC and a $2.1 \%$ improvement in BMD. It also reduced risk of osteoporosis by $45 \%$ in adults whose $\mathrm{Ca}$ and $\mathrm{P}$ intakes were within normal ranges.

\section{Authors' contributions}

AWL participated in the study design and summarizing the data and helped to draft the manuscript. SSC oversaw the entire procedure and helped finalizing the manuscript. Both authors read and approved the final manuscript.

Acknowledgement

The authors thank to Dr. David Hasza for his statistical analysis of the NHANES dataset.

\section{Sponsorship}

This study was supported by nonrestricted research grants of Innophos.

\section{Author details}

${ }^{1}$ NutraSource, Royal Oak, MI, USA. ${ }^{2}$ NutraSource, 6309 Morning Dew Ct., Clarksville, MD 21029, USA.

Received: 14 November 2014 Accepted: 3 March 2015

Published online: 21 March 2015

\section{References}

1. World Health Organization (WHO). 2007. WHO Scientific Group on the Assessmalest of Osteoporosis at Primary Health Care Level. Summary Meeting Report. Brussels, Belgium, 5-7 May 2004. Available at: http://www.who.int/chp/topics/Osteoporosis.pdf.

2. EFSA. EFSA Panel on Dietetic Products, Nutrition and Allergies. Scientific opinion on the substantiation of health claims related to calcium and maintenance of normal bone and teeth (ID 2731, 3155, 4311, 4312, 4703), maintenance of normal hair and nails (ID 399, 3155), maintenance of normal blood LDL-cholesterol concentrations (ID 349, 1893), maintenance of normal blood HDL-cholesterol concentrations (ID 349, 1893), reduction in the severity of symptoms related to the premenstrual syndrome (ID 348, 1892). "cell membrane permeability" (ID 363), reduction of tiredness and fatigue (ID 232), contribution to normal psychological functions (ID 233), contribution to maintenance or achievemalest of a normal body weight (ID 228, 229) and regulation of normal cell division and differentiation (ID237) pursuant to Article 13(1) of Regulation (EC) No 1924/2006. EFSA J. 2010;8:1725. 30 pp.

3. Institute of Medicine (IOM). Dietary Reference Intakes for calcium, phosphorus, magnesium, Vitamin D, and fluoride, vol. 35. Washington, D.C: National Academy of Sciences; 2001. p. 1678-82.

4. Ka“rkkäinen KUM, Lamberg-Allardt CJE. An acute intake of phosphate increases parathyroid hormone secretion and inhibits bone formation in young women. J Bone Miner Res. 1996;11:1905-12.

5. Kemi VE, Kärkkäinen MU, Lamberg-Allardt CJ. High phosphorus intakes acutely and negatively affect $\mathrm{Ca}$ and bone metabolism in a dose-dependent manner in healthy young females. Br J Nutr. 2006;96:545-52.

6. Kemi VE, Kärkkäinen MU, Rita HJ, Laaksonen MM, Outila TA, Lamberg-Allardt CJ. Low calcium:phosphorus ratio in habitual diets affects serum parathyroid hormone concentration and calcium metabolism in healthy women with adequate calcium intake. Br J Nutr. 2010;103:561-8.

7. Kemi VE, Rita HJ, Kärkkäinen MU, Viljakainen HT, Laaksonen MM, Outila TA, et al. Habitual high phosphorus intakes and foods with phosphate additives negatively affect serum parathyroid hormone concentration: a cross-sectional study on healthy premenopausal womales. Public Health Nutr. 2009;12:1885-92.

8. Calvo MS, Kumar R, Heath III H. Persistently elevated parathyroid hormone secretion and action in young women after four weeks of ingesting high phosphorus, low calcium diets. J Clin Endocrinol Metab. 1990;70:1334-40.

9. Metz JA, Anderson JJ, Gallagher Jr PN. Intakes of calcium, phosphorus, and protein, and physical-activity level are related to radial bone mass in young women. Am J Clin Nutr. 1993;58:537-42.

10. Portale AA, Halloran BP, Murphy MM, Morris Jr RC. Oral intake of phosphorus can determine the serum concentration of 1,25-dihydroxyvitamin $D$ by determining its production rate in humans. J Clin Invest. 1986;77:7-12.

11. Spencer $H$, Kramer L, Osis D, Norris C. Effect of phosphorus on the absorption of calcium and on the calcium balance in man. J Nutr. 1978;108:447-57.

12. Heaney RP. Dietary protein and phosphorus do not affect calcium absorption. Am J Clin Nutr. 2000;72:758-61.

13. Heaney RP, Recker RR. Effects of nitrogen, phosphorus, and caffeine on calcium balance in women. J Lab Clin Med. 1982;99:46-55.

14. Rafferty K, Heaney RP. Nutrient effects on the calcium economy: emphasizing the potassium controversy. J Nutr. 2008;138:166S-71. 
15. Heaney RP, Recker RR, Watson P, Lappe JM. Phosphate and carbonate salts of calcium support robust bone building in osteoporosis. Am J Clin Nutr. 2010;92:101-5.

16. Cotton PA, Subar AF, Friday JE, Cook A. Dietary sources of nutrients among US adults, 1994 to 1996. J Am Diet Assoc. 2004;104:921-30.

17. Tucker KL, Morita K, Qiao N, Hannan MT, Cupples LA, Kiel DP. Colas, but not other carbonated beverages, are associated with low bone mineral density in older women: The Framingham Osteoporosis Study. Am J Clin Nutr. 2006;84:936-42.

18. Kemi VE, Kärkkäinen MU, Karp HJ, Laitinen KA, Lamberg-Allardt CJ. Increased calcium intake does not completely counteract the effects of increased phosphorus intake on bone: an acute dose-response study in healthy females. Br J Nutr. 2008;99:832-9.

19. Heaney RP. Phosphorus nutrition and the treatmalest of osteoporosis. Mayo Clin Proc. 2004;79:91-7.

20. Ditscheid B, Keller S, Jahreis $\mathrm{G}$. Cholesterol metabolism is affected by calcium phosphate supplementation in humans. J Nutr. 2005;135:1678-82.

\section{Submit your next manuscript to BioMed Central and take full advantage of:}

- Convenient online submission

- Thorough peer review

- No space constraints or color figure charges

- Immediate publication on acceptance

- Inclusion in PubMed, CAS, Scopus and Google Scholar

- Research which is freely available for redistribution 